\title{
A WHOLE BLOOD SAMPLE-TO-ANSWER LAB-ON-A-CHIP WITH ASYMMETRIC CAPILLARY FORCE BASED BLOOD PLASMA SEPARATOR
}

\author{
Kang Kug Lee* and Chong H. Ahn \\ Microsystems and BioMEMS Laboratory \\ Department of Electrical Engineering and Computing Systems, \\ University of Cincinnati, Cincinnati, Ohio, USA
}

\begin{abstract}
An innovative 'smart' sample-to-answer (S-to-A) polymer lab-on-a-chip (LOC) with asymmetric capillary force based blood/plasma separator has been proposed, developed, and fully characterized for point-of-care clinical testing (POCT) applications. A spray layer-by-layer (LbL) nanoassembly method has been developed and applied for the superhydrophilic surface coated on a cyclic olefin copolymer (COC). Then, the developed superhydrophilic surfaces were designed and optimized for three device applications such as lateral transportation of whole blood in the device by capillary pumping, on-chip whole blood/plasma separation with an asymmetric capillary force, and detection using a capillary-driven lateral flow colorimetric assay. With the integration of the developed three devices, the S-to-A polymer LOC platform has been successfully developed for the colorimetric assay of bovine serum albumin (BSA) from undiluted human whole blood without an external power source.
\end{abstract}

\section{INTRODUCTION}

A blood test is the most common medical diagnostic tool to monitor the health condition of patients who have chronic diseases (such as diabetes) and is of great importance in global health applications. This is because faster or more efficient treatment can be delivered due to the rapid and precise diagnosis of diseases with the human whole blood that contains a massive amount of critical information concerning the function of the body [1]. The blood test is also the best clinical diagnostic for urgent care patients in regions with constrained resources, in the field, or in combat zones. Typical blood tests are performed in a central laboratory which is equipped with bulky and expensive analyzers, and usually require a considerable volume of blood sample, trained personnel, and need relatively long analysis time [2]. Furthermore, the separation of blood plasma from the whole blood is a critical step for most clinical blood analysis based on fluorescence immunoassays to minimize the noise from blood cells interfered with excitation optics. These limitations can be removed with the development of more practical on-chip blood analytical systems.

The microfluidic LOC platform has been developed for the various fields in chemistry, biology, engineering, and biomedical and pharmaceutical research over the past decades [3-6]. With the recent development of microfluidic LOC technology, the miniaturization of laboratory instruments and assays has been realized, leading to lower costs per measurement, reduced sample analysis times, reduced sample volume, and better reproducibility in several applications. Compared with the 96-well based microtiter plate which needs a sample volume of $\sim 100 \mu \mathrm{L}$, the LOC requires a sample of less than $\sim 1 \mu \mathrm{L}$. More importantly, the assay time can also be reduced from several hours to several minutes. The reduction of assay time is mainly due to the short diffusion length that the molecules must travel to immobilize on the solid phase surface because the cross-sectional dimension of a microchannel is around $10 \mu \mathrm{m}$ to $100 \mu \mathrm{m}$ [7]. However, most of the previously reported LOC platforms have limitations in their use because of the need for an external power source in fluid handling such as high-voltage power supplies for the electrokinetic flow, pressure sources such as syringe pumps for the pressure-driven flow, and a mechanical rotator for the centrifugation induced flow $[8,9]$. These instruments are often bulky and expensive, and restrict the use of microchips as an integrated on-chip platform for POCT. Furthermore, at a central laboratory equipped with these instruments, the total turnaround time (TAT) from sample-to-result is extended by the time required for the transportation of sample to the central laboratory. Due to these restrictions, it is very desirable to design and develop a further practical POCT platform for the patients who need an urgent or emergence care.

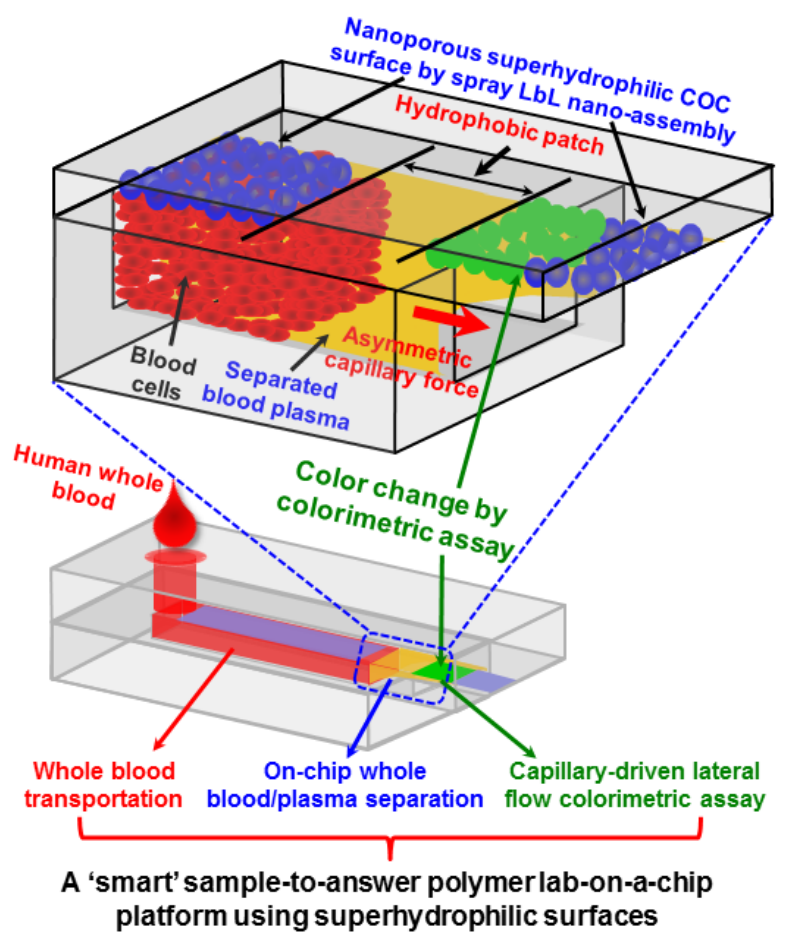

Figure 1: Conceptual diagram for a 'smart' S-to-A polymer LOC platform that simply integrates an on-chip blood/plasma separator with a detection bioassay component without an external power source targeted to POCT applications.

In this work, a new 'smart' whole blood S-to-A polymer LOC platform has been proposed and developed. This platform simply integrates an on-chip blood/plasma separator with a capillarydriven lateral flow colorimetric assay component without an external power source as shown in Figure 1. The developed superhydrophilic surface by a spray LbL nanoassembly method was developed and applied to three specific devices for the lateral transportation of whole blood in the device by capillary pumping, on-chip whole blood/plasma separation with an asymmetric capillary force, and detection using a capillary-driven lateral flow 
colorimetric assay. The developed S-to-A polymer LOC can be a practical platform for POCT applications.

\section{DESIGN AND FABRICATION}

\section{Microfabrication of S-to-A Polymer LOC Platform}

Figure 2 summarizes the fabrication process of the S-to-A polymer LOC platform integrated with an on-chip blood/plasma separator. A CNC milling machine was used for fabricating a master mold. The $\mathrm{CNC}$ milling machine manufactures various sizes of structures in the range of $\mu \mathrm{m}$ to $\mathrm{mm}$ in scale. The microchannel was designed to transport the introduced whole blood, measure the separated volume of plasma from whole blood, and to characterize the asymmetric capillary movement of blood plasma for colorimetric assay. The volume of the inlet reservoir was approximately $3 \mu \mathrm{L}$, which is comparable to the volume of whole blood taken from a finger prick using a commercial device.

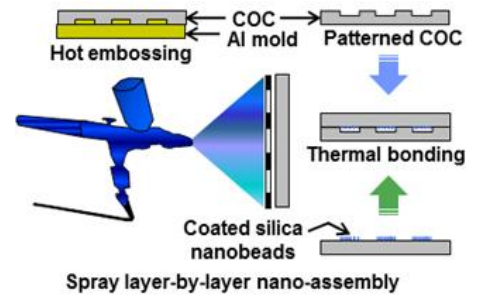

(a)

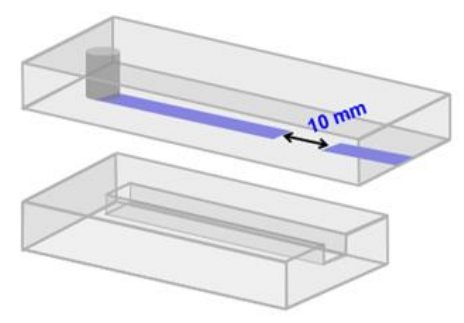

(c)

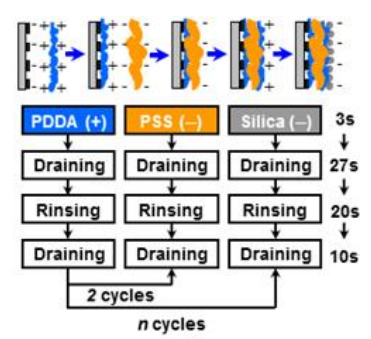

(b)

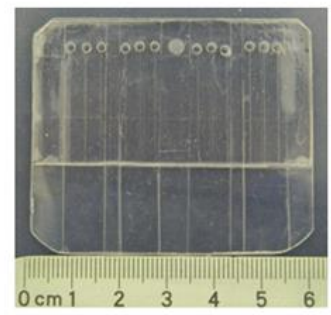

(d)
Figure 2: Summary of fabrication process of the S-to-A polymer LOC device.

Then, COC substrates were patterned with microfluidic channels using a hot embossing technique for the spray $\mathrm{LbL}$ coating process. After this, the spray LbL nanoassembly process with silica nanoparticles [10] was performed for the asymmetric superhydrophilic surface with a $10 \mathrm{~mm}$ hydrophobic patch over the COC microchannel. Finally, the COC substrate with microchannels was bonded with the spray functionalized COC substrate by a thermoplastic fusion bonding technique using an embossing machine [11]. The designed microchannel with $400 \mu \mathrm{m}$ $\times 100 \mu \mathrm{m} \times 50 \mathrm{~mm}(\mathrm{~W} \times \mathrm{D} \times \mathrm{L})$ and the $10 \mathrm{~mm}$ hydrophobic patch are shown in Figure 2(c). The fabricated S-to-A polymer LOC device is shown in Figure 2(d).

\section{Preparation of Colorimetric Assay for Protein Quantification}

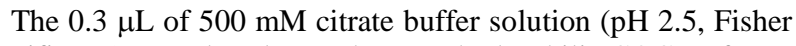
Scientific, IL) was placed onto the superhydrophilic COC surfaces, followed by the layered $0.3 \mu \mathrm{L}$ of $3.3 \mathrm{mM}$ of tetrabromophenol blue (TBPB, Sigma-Aldrich, St. Louis, MO) in 95\% ethanol (Fisher Scientific, IL) shown in Figure 3(b). The reagents on the superhydrophilic surface were allowed to dry at $25{ }^{\circ} \mathrm{C}$ for $10 \mathrm{~min}$.
Introducing the spiked whole blood with BSA into the S-to-A polymer LOC device, the blood plasma successfully separated from the whole blood was transferred to the detection zone for colorimetric assay described in Figure 3(d). This colorimetric protein assay uses the nonspecific binding of TBPB to proteins. The reaction is through a combination of electrostatic (sulfonate) and hydrophobic (biaryl quinone methide) interactions [12]. When reacted, the phenol in TBPB deprotonates and the color of the dye shifts from yellow to blue, which indicates the presence of proteins in separated blood plasma $[12,13]$. This colorimetric protein assay was calibrated and characterized with spiked concentrations ranging from ranging from $0-5 \mathrm{mg} / \mathrm{mL}(0 \mu \mathrm{M}$ to $75 \mu \mathrm{M})$ of BSA, which is clinically relevant range [14].

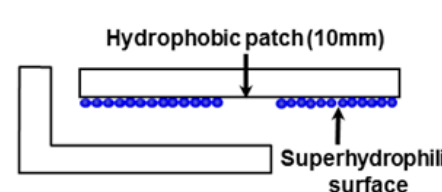

(a)

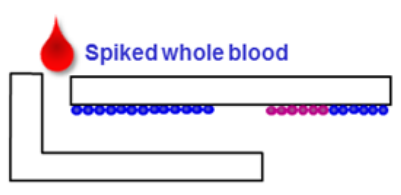

(c)

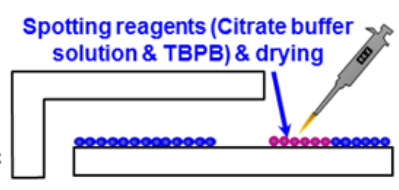

(b)

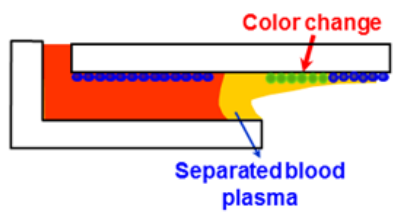

(d)
Figure 3: Preparation of colorimetric assay for protein quantification in a S-to-A polymer LOC platform.

\section{EXPERIMENTAL RESULTS}

\section{Capillary-driven Lateral Flow Colorimetric Assay}

Figure 4 shows a 'smart' S-to-A polymer LOC platform that integrates an on-chip blood/plasma separator with capillary-driven lateral flow colorimetric assay. The reagents for colorimetric assay were placed onto the patterned superhydrophilic surfaces, which were modified by the spray LbL nanoassembly depicted in Figure 4(a). The BSA concentration for spiking in whole blood was measured first using an analyzer in laboratory and then used as a background reference before spiking. Single droplet of $3.0 \mu \mathrm{L}$ human whole blood, a suitable amount for disposable single-use platform for POCT, spiked with different concentrations of BSA solution were injected into the inlets of the S-to-A LOC device shown in Figure 4(b). When the mixture of whole blood and BSA solution was injected into the inlet of the device, the top superhydrophilic surface generated capillary flow of whole blood through the microchannel. When the mixture encountered the 10 $\mathrm{mm}$ hydrophobic patch region, the flow of the whole blood was effectively retarded for a short period of time $(<1 \mathrm{~min})$. This retarding effect caused a continuous selective accumulation of blood cells within the hydrophobic patch region. The movement of the blood cells were effectively reduced, allowing the blood plasma to move forward due to the differences in flow velocity between the cellular component and the blood plasma over the patch region. Thus, the blood plasma was successfully separated from the whole blood throughout this accumulated column of blood cells, a so-called 'self-built-in blood cell microfilter' [15]. The color changes from yellow to blue were obtained in Figure 4(b). The intensity of the blue color was increased depending on the concentrations of BSA spiked in whole blood. Figure 4(c) 
shows a magnified image of moving separated blood plasma. This picture demonstrates the filtering efficiency by asymmetric capillary force and the patterned hydrophobic patch.

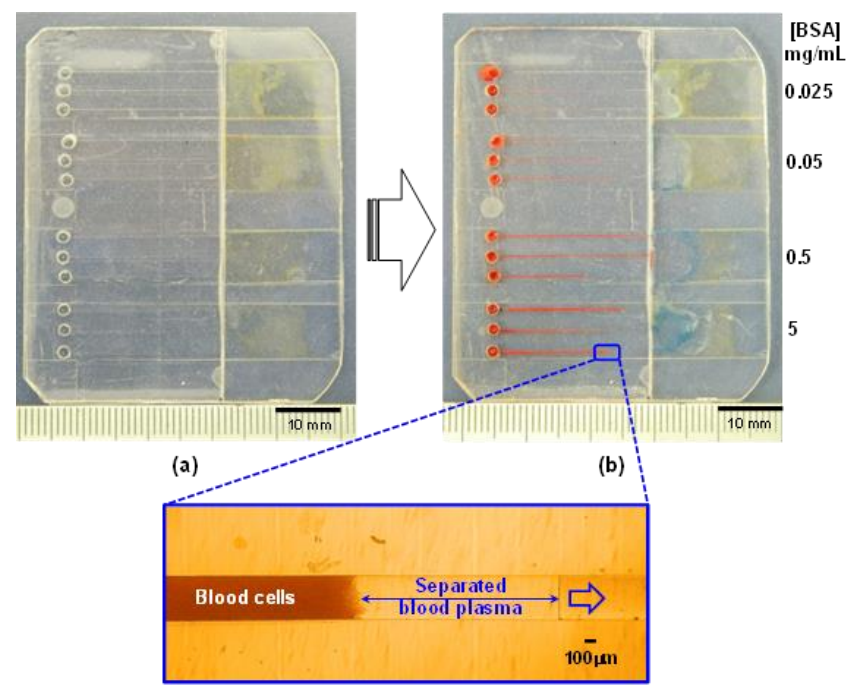

(c)

Figure 4: Capillary-driven lateral flow colorimetric assay in a $S$ to-A polymer LOC device.

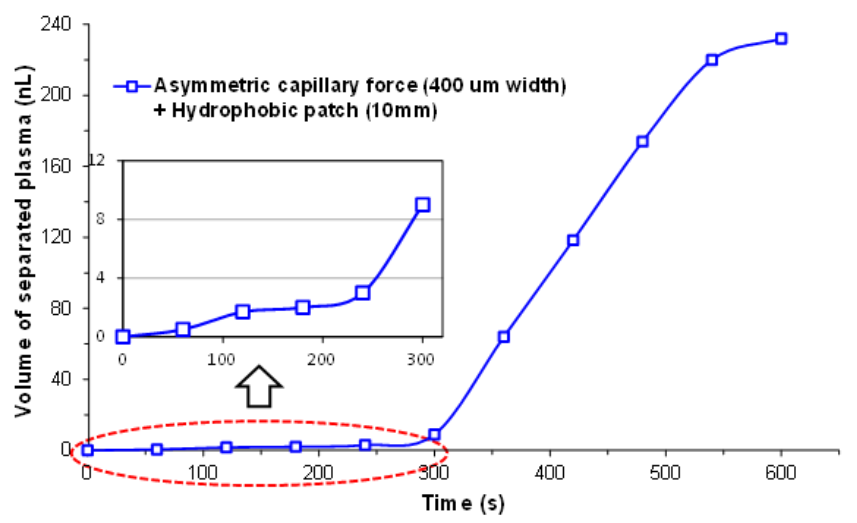

Figure 5: Volume of the separated plasma $(n L)$ over time in the $S$ to-A polymer LOC platform; $400 \mu \mathrm{m}$ width microchannel with a 10 mm hydrophobic patch.

The movement of the separated plasma was monitored with an optical microscope by using reference marks along the microchannel. The volume of separated plasma was obtained by measuring the length of the leading edges between separated blood plasma and blood cells over time. The blood plasma shown in Figure 4(c) was successfully separated through a $5.8 \mathrm{~mm}$ long microchannel, where its cross-sectional area was $400 \mu \mathrm{m} \times 100$ $\mu \mathrm{m}(\mathrm{W} \times \mathrm{D})$. A volume of $232 \mathrm{~nL}$ of separated plasma from a single droplet $(3.0 \mu \mathrm{L})$ of whole blood was finally obtained in a microchannel as shown in Figure 5. The summated total volume of the separated plasma from three microchannels in the device was used for blood analysis using capillary-driven lateral flow colorimetric assay. The summated volume of the separated plasma was large enough for on-chip POCT with undiluted human whole blood. The volume of the separated plasma below 300 seconds in Figure 5 was slightly increased over time due to the accumulation of blood cells over the hydrophobic patch during the separation. The volume of the separated plasma was dramatically increased after 300 seconds due to strong asymmetric capillary force throughout the 'self-built-in blood cell microfilter' developed in a $10 \mathrm{~mm}$ hydrophobic patch.

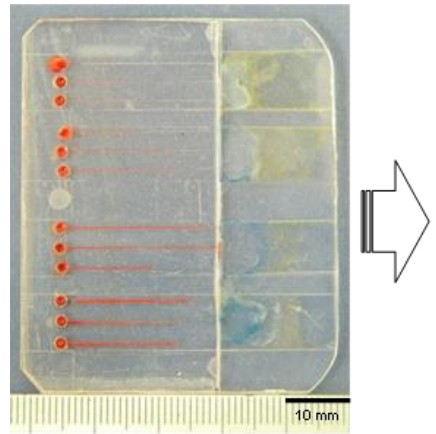

(a)

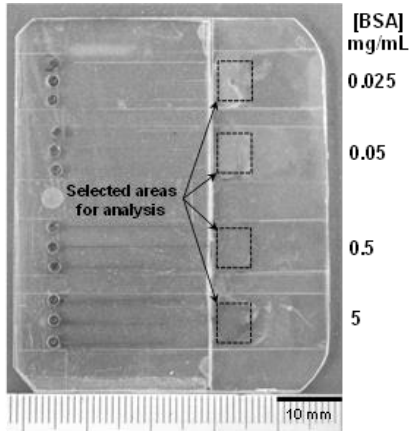

(b)

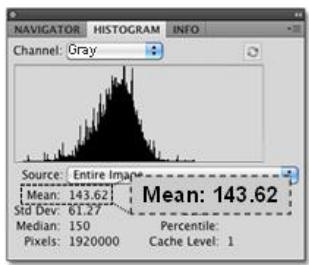

(c)

Figure 6: Procedure for quantifying protein levels in separated blood plasma through a S-to-A polymer LOC device using Adobe Photoshop software.

\section{Calibration Curve for Colorimetric Assay with Spiked Proteins}

In clinical point of view, the quantification of proteins in human whole blood is significant in the diagnostics of various diseases. The variations in protein level in blood plasma or serum separated from whole blood is attributed from a variety of diseases such as blood disorders, digestive disorders, kidney disorders, and cancers [16]. In this assay, the concentration of BSA spiked in whole blood was successfully quantified by the S-to-A LOC device using capillary-driven lateral flow colorimetric assay. TBPB ionizes and binds with proteins to cause a color change. A positive result is indicated by a color change from yellow to blue. The intensity of the blue color is proportional to the amount of the proteins spiked in blood plasma or whole blood. To generate the calibration curve from the colorimetric assay in the device, the same method used in paper-based colorimetric assay was accomplished [17]. The images of the color changes were captured using a digital camera and the intensity was obtained with Adobe Photoshop in a gray scale mode (Figure 6). The mean values of the selected area through Adobe Photoshop software correlate with the concentration of the spiked proteins in Figure 6(c).

The intensity of color change was quantified by a calibration curve in terms of the protein concentrations in Figure 7. The regression equation is Intensity $=8.1348 \ln (x)+54.405\left(R^{2}=\right.$ 0.8958 ), where $x$ is the concentration of protein (in $\mathrm{mg} / \mathrm{mL}$ ). The colorimetric assay was calibrated with blood plasma samples 
separated from three microchannels with known protein concentrations ranging for $0-5 \mathrm{mg} / \mathrm{mL}(0 \mu \mathrm{M}$ to $75 \mu \mathrm{M})$ of BSA. The limit of detection was $25 \mu \mathrm{g} / \mathrm{mL}$, which is better than that from paper-based microfluidic device or conventional method [17-19]. For improving of the detection limit, the assay need to be further optimized or the exploration of new assay methods is desirable. Both blood plasma separation and quantitative analysis in whole blood was simultaneously demonstrated in a S-to-A polymer LOC platform that integrates an on-chip blood/plasma separator with capillary-driven colorimetric assay.

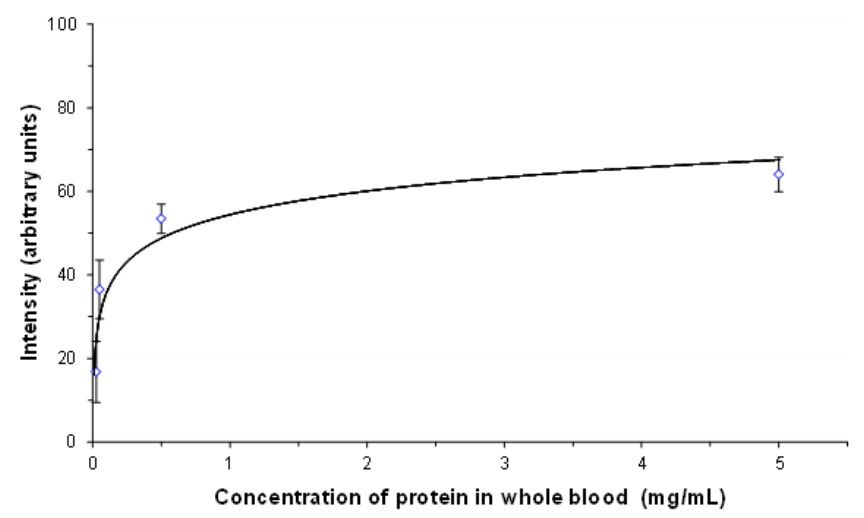

Figure 7: Quantification of protein concentration in human whole blood samples using a S-to-A polymer LOC device with integrated on-chip blood/plasma separator. The limit of detection was $25 \mu \mathrm{g} / \mathrm{mL}$.

\section{CONCLUSION}

In conclusion, the newly developed superhydrophilic surfaces were applied for the realization of a capillary-driven lateral flow colorimetric assay platform targeted to a 'smart' S-to-A polymer LOC device. Both high-quality blood plasma separated from whole blood and its quantitative analysis were simultaneously demonstrated in the S-to-A polymer LOC platform. The limit of detection (LoD) in the S-to-A polymer LOC platform for BSA was comparable to or better than that of the paper-based colorimetric assay. The developed S-to-A polymer LOC device is a new platform for the POCT. There is still a plenty of room for the improvement of the LoD by the optimization of the assay or adoption of other assay methods. The developed S-to-A polymer LOC can be a practical platform for POCT applications.

\section{REFERENCES}

[1] M. Toner and D. Irimia, "Blood-on-a-chip," Annu. Rev. Biomed. Eng., 7, 77 (2005).

[2] D. Mabey, R. W. Peeling, A. Ustianowski, and M. D. Perkins, "Diagnostics for the developing world," Nat. Rev. Microbiol., 2, 231 (2004).

[3] P. A. Auroux, D. Iossifidis, D.R. Reyes, and A. Manz, "Micro total analysis systems: Analytical standard operations and applications," Anal. Chem., 74, 2637 (2004).

[4] S. K. Sia, V. Linder, B. A. Parviz, A. Siegel, and G. M. Whitesides, "An integrated approach to a portable and low cost immunoassay for resource-poor settings," Angew. Chem., Int. Ed., 43, 498 (2004).

[5] H. Craighead, "Future lab-on-a-chip technologies for interrogating individual molecules," Nature, 442, 387 (2006).
[6] J. El-Ali, P. K. Sorger, and K. F. Jensen, "Cells on chips," Nature, 442, 403 (2006).

[7] S. Cesaro-Tadic, G. Dernick, D. Juncker, G. Buurman, H. Kropshofer, B. Michel, C. Fattinger, and E. Delamarche, "High-sensitivity miniaturized immunoassays for tumor necrosis factor a using microfluidic systems," Lab Chip, 4, 563 (2004).

[8] P. Mitchell, "A perspective on protein microarrays," Nat. Biotechnol., 20, 225 (2002).

[9] A. Ulman, "Formation and structure of self-assembled monolayers," Chem. Rev., 96, 1533 (1996).

[10] K. K. Lee and C. H. Ahn, "Superhydrophilic Multilayer Silica Nanoparticle Networks on a Polymer Microchannel Using a Spray Layer-by-Layer Nanoassembly Method," ACS Applied Material \& Interfaces, 5(17), 8523 (2013).

[11] C. H. Ahn, J. W. Choi, G. Beaucage, J. H. Nevin, J. B. Lee, A. Puntambekar, and J. Y. Lee, "Disposable smart lab on a chip for point-of-care clinical diagnostics," Proceedings of the IEEE, 92, 154 (2004).

[12] W. Cao, Q. Jiao, Y. Fu, L. Chen, and Q. Liu, "Mechanism of the interaction between bromophenol blue and bovine serum albumin," Spectrosc. Lett., 36, 197 (2006).

[13] J. D. Jr. Peele, R. H. Gadsden, and R. Crews, "Semiautomated vs. visual reading of urinalysis dipsticks," Clin. Chem., 23, 2242 (1997).

[14] A. W. Martinez, S. T. Phillips, M. J. Butte, and G. M. Whitesides, "Patterned paper as a platform for inexpensive, low-volume, portable bioassays," Angew. Chem., Int. Ed., 46, 1318 (2007).

[15] K. K. Lee and C. H. Ahn, "A new on-chip whole blood/plasma separator driven by asymmetric capillary forces," Lab Chip, 13, 3261 (2013).

[16] A. J. Ninfa, D. P. Ballou, and M. Benore, Chapter 4: Quantification of Protein Concentration, Fundamental Laboratory Approaches for Biochemistry and Biotechnology, $2^{\text {nd }}$ Ed., Wiley, 2009.

[17] A. K. Yetisen, M. S. Akram, and C. R. lowe, "Paper-based microfluidic point-of-care diagnostic devices," Lab Chip, 13, 2210 (2013).

[18] W. Wang, W.-Y. Wu, and J.-J. Zhu, "Tree-shaped paper strip for semiquantitative colorimetric detection of protein with self-calibration," J. Chromatogr. A, 1217(24), 3896 (2010).

[19] W. Dungchai, O. Chailapakul, and C. S. Henry, "Use of multiple colorimetric indicators for paper-based microfluidic devices," Anal. Chim. Acta, 674, 227 (2010).

\section{CONTACT}

*Kang Kug Lee, tel: +1-513-535-9030; Leek8@ mail.uc.edu 